\title{
LDMA: Load Balancing Using Decentralized Decision Making Mobile Agents
}

\author{
M. Aramudhan ${ }^{1}$ and V. Rhymend Uthariaraj ${ }^{2}$ \\ ${ }^{1}$ Research scholar, Dept.of. IT, MIT, Anna University, Chennai-25, Tamilnadu, India \\ aranagai@yahoo.co.in \\ ${ }^{2}$ Professor, Dept.of. IT, MIT, Anna University, Chennai-25, Tamilnadu, India \\ rhymendeannauniv.edu
}

\begin{abstract}
This paper introduces a new load balancing algorithm, called LDMA (Load balancing using Decentralized decision making Mobile Agents), which distributes load among clustered web servers connected in a mesh topology, by a communications network and compares its performance with other load balancing algorithm: MALD (Mobile Agent based LoaD balancing). Architecture is developed for the same and all necessary attributes such as load deviation, system throughput and response time incurred as a result of the work are dealt with. In past works, a centralized decision making algorithm was used for dispatching requests to web servers in the distributed client/server environment. In the proposed approach, a decentralized decision making algorithm is used for distributing requests among web servers. The simulator is developed in $\mathrm{C}++$ and its performance is evaluated. The analysis shows that LDMA is better than centralized decision making load balancing algorithms.
\end{abstract}

Keywords: Load balancing, decentralized decision making, mobile agents, clustered web servers.

\section{Introduction}

A distributed computer system is a collection of self-sufficient computers located at diverse or identical sites and associated by a communication network. The performance of a distributed system is enhanced to an adequate level by distributing the workload among the servers. Normally, load balancing occurs at the server side and assists to balance the load in distributed computer system. Winston [1] proved that the most excellent mechanism for achieving optimal response time is to distribute the workload equally among the servers. Incoming client requests should be evenly distributed among the servers to achieve quick response time. Traditional load balancing approaches on distributed web servers are implemented based on message passing paradigm. At present, mobile agent technology is used to implement load balancing on distributed web servers. Mobile agent is defined as a software component that can move freely from one host to another on a network and transport its state and code from home host to other host and execute various operations on the site [6]. The mobile agent based approaches have the merit of high flexibility, low network traffic and high asynchrony. 
Distributed web servers deploy in different geographical scopes. They can be organized into cluster of web servers linked through Local Area Network (LAN), to provide high processing power and reliability. The servers are heterogeneous in terms of hardware configuration, operating systems and processing power. Generally, load balancing on Wide Area Network (WAN) is more time consuming since it involves the interaction between remote servers for gathering load information, negotiating on load reallocation and transporting the workload [2]. All approaches in this context so far has been using only centralized decision making. But, an architecture based purely on a centralized server is extremely vulnerable to congestion. In addition, it introduces a single point of failure in the Web system, as stated in [9]. Hence, we approach the problem in a totally different dimension, by introducing the concept of "decentralized decision making". LDMA uses mobile agents for this idea. In LDMA, there is no collection of load information and request transfer policy between web servers. Each server processes client requests independently and interact with others to share the workload.

\section{The LDMA Framework}

The overall architecture of the LDMA framework is as shown in figure 1. The LDMA framework defines two worlds, namely: client world and server world. The client world is an aggregation of all the clients in the physical world, and the server world is an aggregation of the clustered web servers, which are called replicas. The client world communicates with the server world via the dispatcher. The queue at the dispatcher has a finite buffer and a tail drop discard policy. But, unlike the other approaches, in which the dispatcher re-routes the client requests to corresponding servers (centralized decision making), the work of the LDMA dispatcher is just to broadcast client requests to all the replicas. The decision-making for load balancing among replicas take place only by the interaction of mobile agents between the replicas. The replicas are inter-connected by mesh topology. Each replica has the following two modules:

1. MASM - Mobile Agent Servicing Module

2. SND - Search aNd Destroy module.

The work of the MASM is to communicate the mobile agents with other replicas to make them decide which replica may process a request and the work of the SND module is to search for and delete (remove) a particular request from the replicas queue. The LDMA framework uses the concept of "ranked web-servers", i.e., each replica is statically assigned a rank based on which priority is given for processing a request.

\subsection{LDMA Load Balancing Scheme}

Initially, upon the arrival of a client request, the dispatcher broadcasts it to all the replicas, after assigning a RID (Request ID) to it. The replicas accept the request, but the request processing does not start immediately. Instead, the request is placed in its 


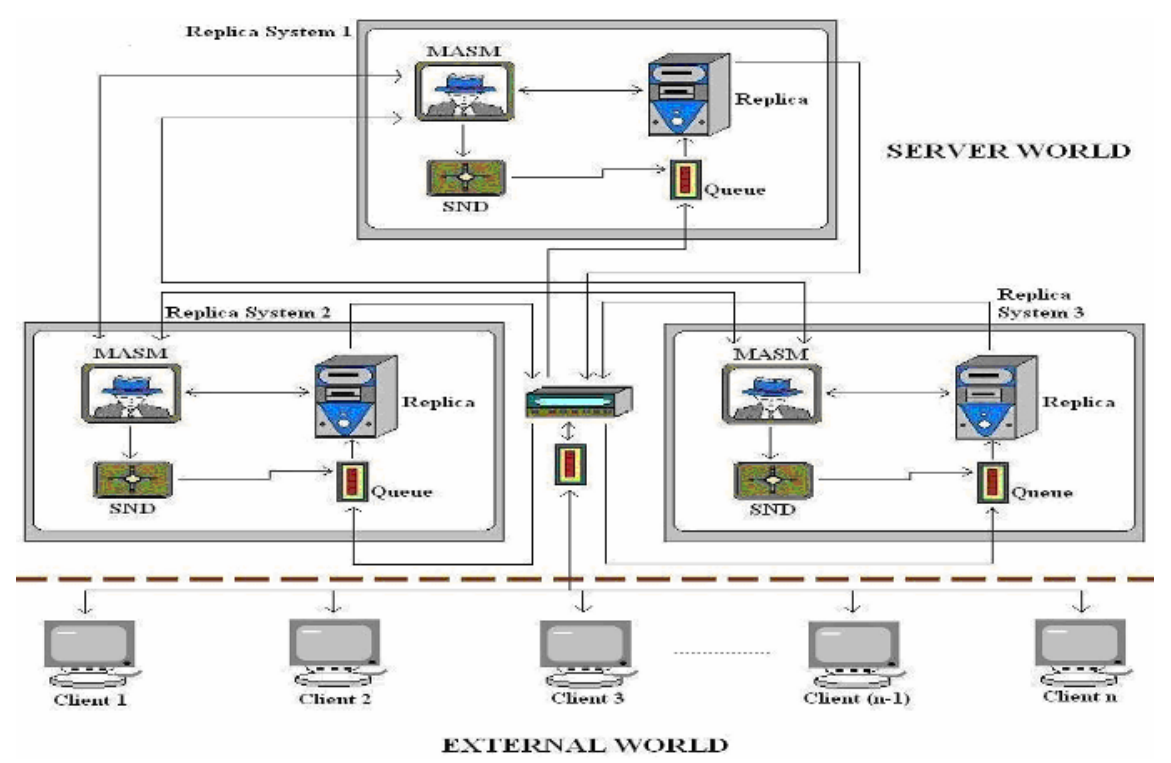

Fig. 1. The LDMA Framework

"waiting state". Each replica sends a mobile agent, with replica's rank and the RID just accepted, which we call "RID under siege". The mobile agents travel to the other replicas and check the state of the same request in the destination replicas. Then, they can return back to the source replica with either of the following messages:

1. Accepted: This case occurs when the rank of source replica is less than the rank of the destination replica, and the RID under siege is in waiting state in the destination replica. On receiving back this message, the source replica just ignores the accepted request, and chooses the next request.

2. Deleted: This case occurs,

i. when the rank of source replica is greater than the rank of the destination replica and the RID under siege is in waiting state in the destination replica, or

ii. irrelevant of the ranks, the RID under siege is in the queue in the destination replica.

The mobile agent triggers the SND module at the destination replica, which removes the RID under siege from the destination replica. On receiving back this message, the source replica starts processing the request.

3. Not Found: A mobile agent returns back to source replica, with this message, when RID under siege is not found either in its waiting state or even at the queue of the destination replica. This case occurs when the RID under siege has already been removed from the destination replica's queue, by a mobile agent from the other replica. On receiving back this message, the source replica may choose to ignore or accept the request, depending on the other mobile agent's response. 


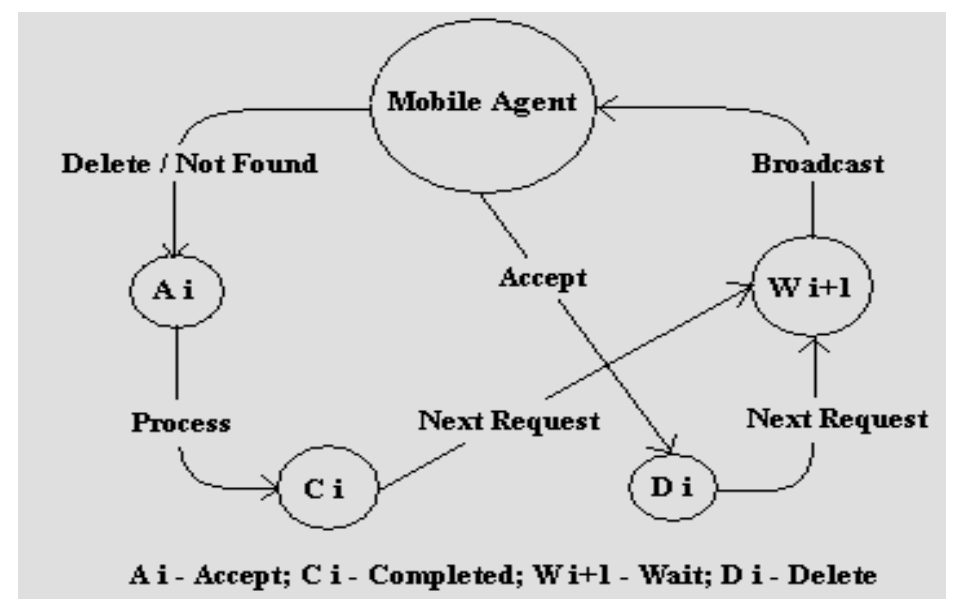

Fig. 2. LDMA Transition Diagram

To retrospect (as shown if figure 2), a replica, on accepting a request, sends mobile agents to other replicas and waits for the response. It ignores the request, if at least one of the responses is an "Accepted" message. It starts processing the request otherwise. Moreover, in case of a packet loss of a mobile agent, a replica waits for a maximum of twice the RTT (Round Trip Time) of the mobile agent. In case of no response message, the replica starts processing the request. Also, the dispatcher assigns RIDs using mod $\mathrm{N}$ arithmetic, i.e.,

$$
\mathrm{RID}=\mathrm{i} \bmod \mathrm{N}, \text { where } \mathrm{i}=0,1,2,3 \ldots
$$

The RIDs are in increasing order. Hence, the work of SND module at a replica is easier and it searches for RID under siege from the top (using the sequential search algorithm), till ith request in the queue, where i is "just greater than" the RID under siege. After the ith request, the RID under siege cannot be found elsewhere in the queue (since RIDs are in increasing order), except in the next cycle of RIDs.

\section{LDMA Simulation Model}

The software simulator was designed in $\mathrm{C}++$ and implemented to model the LDMA load balancing technique in the distributed web server environment. Workload of a replica is determined by the number of request processed at each replica. To achieve best performance results a method applied needs to minimize workload difference between the replicas. For load balancing algorithm $T_{e}$ and $T_{d}$ are complied. $T_{e}$ is the elapsed time from the start of the first client call until the entire clients call and $T_{d}$ is the delay time representing the sum of all the delays associated with the client's requests. Simulation parameters governing the generation of client's events are summarized below: 
Load distribution: The load on the server is denoted by the number of requests processed in the server. The average load distribution deviation over all servers is calculated to show the effect of load balancing.

System throughput: the overall throughput of the web server cluster, measured in the number of requests processed per second.

Network traffic: the overall communication overhead in the cluster, measured in the total number of data (bytes) transferred in the communication.

Table 1. Simulation parameters used

\begin{tabular}{|l|c|}
\hline Simulation Parameter & Value \\
\hline Servers & 3 \\
\hline Request/client & 1 \\
\hline $\begin{array}{c}\text { Data rate (Transmission } \\
\text { speed) }\end{array}$ & $10 \mathrm{MBps}$ \\
\hline http request file size & $<=2 \mathrm{MB}$ \\
\hline Propagation Delay & Negligible \\
\hline Mobile agent RTT & $0.5 \mathrm{~ms}$ \\
\hline Processing Delay & Negligible \\
\hline
\end{tabular}

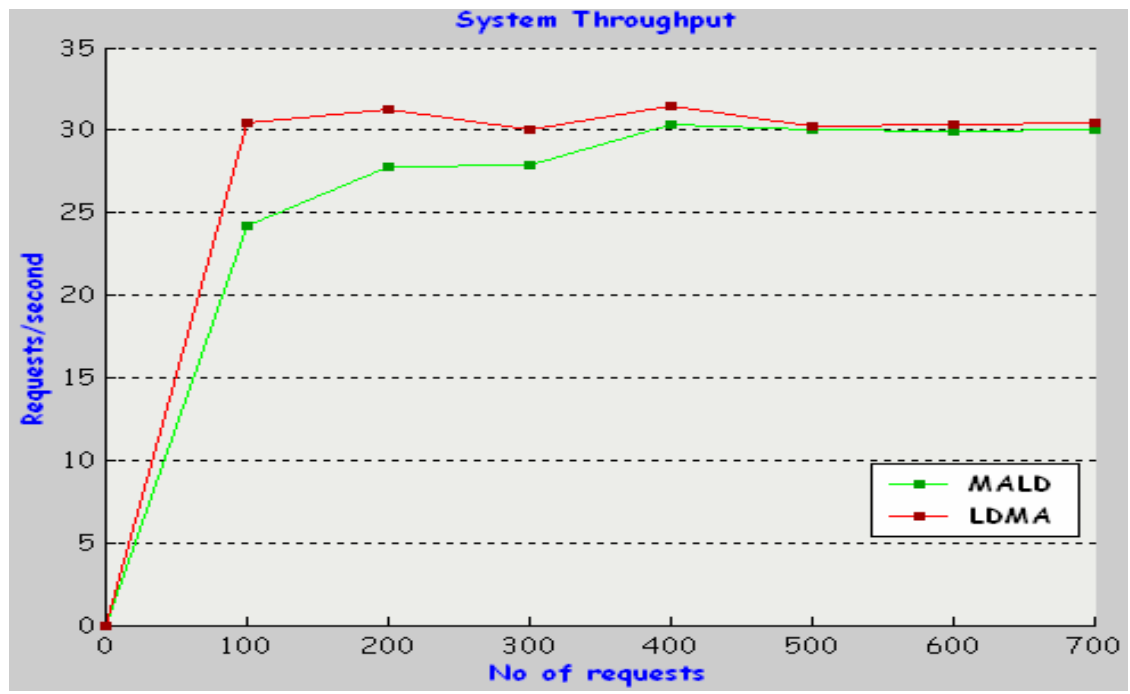

Graph 1. The LDMA system throughput 


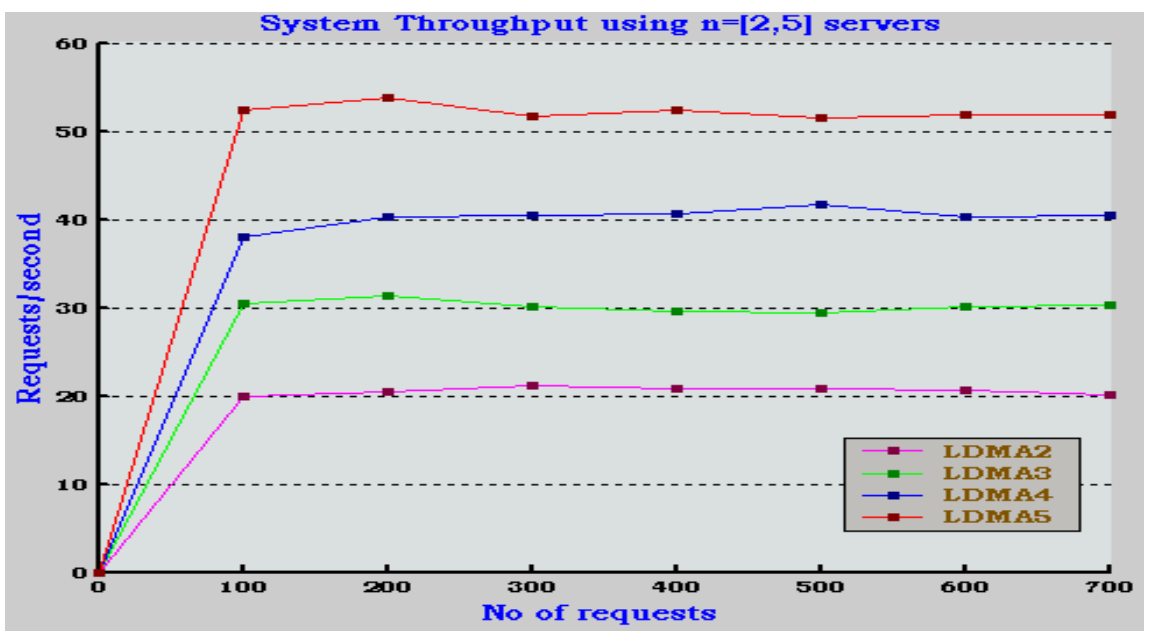

Graph 2. System throughput with web server 2,3,4,5

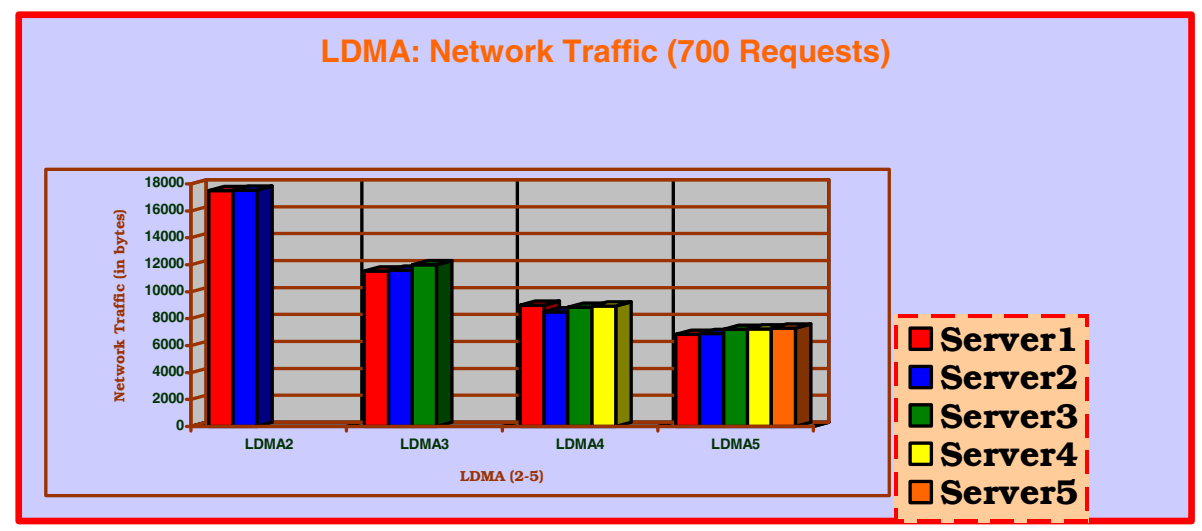

Graph 3. LDMA Network Traffic

Graph-1 shows the system throughput performance of MALD and LDMA for 700 requests. The LDMA performance is slightly better than MALD. Graph-2 shows the system throughput of LDMA having different number of servers in cluster. Graph 3 shows the network traffic of LDMA. The overhead of the packet is 50 bytes. The dispatcher broadcast the incoming requests to all servers. The communication overhead for totally $\mathrm{N}$ servers is as high as $\mathrm{O}\left(\mathrm{N}^{2}\right)$. Network traffic is measured by the total number of bytes transferred in the communication. The network traffic of web server cluster having 2 to 5 servers is as shown in Graph-3. In the beginning, three server's exchanges message for processing the request takes 300 bytes communication overhead. The next two requests take 200, 100 bytes communication overhead respectively. The communication overhead of the afterward requests depends on the previous request processing time. 
Table 2. Load distribution on three servers

\begin{tabular}{|c|c|c|c|c|c|c|c|c|c|c|}
\hline \multirow{6}{*}{ LDMA } & \multicolumn{2}{|c|}{ Total no of request } & 160 & 202 & 188 & 207 & 172 & 143 & 84 & 115 \\
\hline & \multirow{3}{*}{$\begin{array}{c}\text { Reques is } \\
\text { serner }\end{array}$} & Severl & 48 & 71 & 67 & 67 & $s$ & 45 & 24 & 3 \\
\hline & & Sever? & 57 & 66 & 60 & 68 & 57 & 5 & 20 & 3 \\
\hline & & Sener 3 & 5 & 65 & 61 & 72 & 57 & 45 & 31 & 3 \\
\hline & \multicolumn{2}{|c|}{ Average deviation } & 3.56 & 7.33 & 2.89 & 2.0 & 0.44 & 3.56 & 2.67 & 0.44 \\
\hline & \multicolumn{2}{|c|}{$\begin{array}{l}\text { Onerall average } \\
\text { deviation }\end{array}$} & \multicolumn{8}{|c|}{2.86} \\
\hline \multirow{6}{*}{ MALD } & \multicolumn{2}{|c|}{ Total no of request } & 160 & 202 & 188 & 207 & 172 & 143 & 806 & 115 \\
\hline & \multirow{3}{*}{$\begin{array}{c}\text { Reques }: ~ \\
\text { server }\end{array}$} & Senerl & 65 & 6 & 37 & 74 & 68 & 42 & 30 & 30 \\
\hline & & Server? & 5 & 79 & 76 & 5 & 48 & 50 & 37 & 34 \\
\hline & & Server3 & 37 & 00 & 75 & 78 & 56 & $s 1$ & 166 & 48 \\
\hline & \multicolumn{2}{|c|}{ Average deviation } & 10.89 & 7.78 & 17.11 & 9.30 & 7.11 & 3.78 & 7.51 & 6.44 \\
\hline & \multicolumn{2}{|c|}{$\begin{array}{l}\text { Orerall average } \\
\text { deviation }\end{array}$} & \multicolumn{8}{|c|}{874} \\
\hline
\end{tabular}

\section{Conclusion}

LDMA approach to load balancing possesses several advantages. First, decision making is decentralized and response time improves as the number of replicas increase. Second, use of mobile agents imposes the merits of high flexibility, low network traffic and high asynchrony. Third, no replica remains idle at any time while other replicas are busy processing requests. The requests start processing in the arrival order. But still, this method has some drawbacks. First, the use of mesh topology to inter-connect the replicas, limits the scalability of the system to a certain extent. But, since usually web server clusters in LAN consist of a maximum of only 7 to 8 servers, the system is considered to be scalable. Second, a failure or fault in the transaction path of mobile agents between two replicas may result in processing of the same request by many replicas and hence reduce the throughput of the entire system. But, this kind of fault is very rare in a LAN environment and is also easy to detect and repair.

\section{References}

1. W. Winston: Optimality of the Shortest Line Discipline. Journal of Applied Probability (1977) 17-28.

2. Jiannong cao, Yudong Sun, Xianbin Wang and Sajal K. Das :Scalable:Load Balancing on Distributed Web Servers Using Mobile Agents. Journal of Parallel and Distributed Computing, Vol.63, Issue 10. (2003) 996-1005.

3. Huamin Chen and Arun Iyengar :A Tiered System for Serving Differentiated Content. Journal of World Wide Web, Vol.6, Issue 4. (2003) 331-352.

4. Lang fang, Aleksander Slominski and Dennis Gannon: Web Services Security and Load Balancing in Grid Environment. Proc.of. International Conference on Grid Computing, Las Vegas, June (2005). 
5. Gianfranco ciardo, Almariska and Evgenia smirni: EQUILOAD: a Load Balancing Policy for Clustered Web Servers. Proc. of Parallel and Distributed systems (2004)14201425.

6. Altec software business unit (2004), "Mobile Agents System for the Interconnection of Working Groups. Interconnection network vol.5 (2), (2004) 181-191.

7. Foundry Networks, White Paper -Server Load Balancing in Today's Web-Enabled Enterprises, (2002).

8. Reinhardtriedl:Workload Modeling for Load Balancing in Distributed DB/DC Transaction Processing (1999).

9. Marco Conti, Enrico Gregori and Fabio Panzieri :Load Distribution among Replicated Web Servers: A QoS-based Approach. (1999).

10. Baruch Awerbuch, Mohammad T.Hajiaghayi, Robert D.K leinberg and Tom:Online Client-Server Load Balancing without Global Information. in proc.of the sixteenth annual ACM-SIAM symposium on Discrete Algorithms(2005) .

11. Morharchol-Balter, Bianca Schroeder, Nikhil Bansal, and Mukesh Agrawal: Size-Based Scheduling to Improve Web Performance, in ACM Transactions on Computer Systems, Vol. 21, No. 2. (2003)

12. Milan E.Soklic: Simulation of Load Balancing Algorithms: A Comparative Study. in SIGCSE Bulletin vol.34, No.4, Dec. (2002). 